\title{
Die überbewertete Hypoxämie
}

\section{Köhler}

\section{Overestimation of Hypoxemia}

\section{Zusammenfassung}

Bei Extrembergsteigern wie auch bei Patienten mit stabiler schwerer ventilatorischer Insuffizienz (z.B. Obesitas-Hypoventilations-Syndrom, Skoliose) erleben wir Zustände schwerster Hypoxämie mit milder oder nur geringer subjektiver Beeinträchtigung. Auch Organschäden werden dabei nicht beobachtet. Auf der anderen Seite wissen wir aber aus den gut dokumentierten Studien zur Sauerstofflangzeittherapie, dass bei Hypoxämie infolge COPD mit Werten von $\mathrm{pO}_{2}$ unter $55 \mathrm{~mm} \mathrm{Hg}$ die Lebenserwartung durch die Sauerstoffgabe etwa verdoppelt wird.

Dieser Widerspruch löst sich auf, wenn man das Ausmaß der Ventilation berücksichtigt, dessen integraler klinischer Wert das $\mathrm{pCO}_{2}$ darstellt. Die in der Sauerstofflangzeittherapie untersuchten Patienten hatten alle eine mehr oder weniger ausgeprägte Hyperkapnie infolge überlasteter Atempumpe. Sauerstoffgabe reduziert die Ventilation (sichtbar an Zunahme der Hyperkapnie) und entlastet damit die Atempumpe. Nachfolgestudien zur Sauerstofflangzeittherapie haben gezeigt, dass das Ausmaß einer stabilen Hyperkapnie (trotz vorhandener Hypoxämie) positiv mit der Lebenserwartung korreliert ist. In dieser Übersicht wird der pathophysiologische Hintergrund dazu diskutiert. Zentrale Regelgröße für die Sauerstoffversorgung des Organismus ist nicht der Sauerstoffpartialdruck, sondern der Sauerstoffgehalt, der multipliziert mit dem Herzminutenvolumen das Sauerstoffangebot bestimmt. Durch zahlreiche Regulationsfaktoren (z.B. Polyglobulie oder Expression von sauerstoffresistenten Isoenzymen der Atmungskette) kann der Organismus die mit der Hypoventilation verbundene Hypoxämie so gut kompensieren, dass keine organgefährliche Hypoxie daraus resultiert. Diese pathophysiologische Erkenntnis hat unmittelbare Auswirkungen für die Intensivmedizin, die üblicherweise den Sauerstoffpartial-

\section{Abstract}

Extreme mountain climbers and patients with stable but severe ventilatory insufficiency (e.g. obesitas hypoventilation-syndrome, scoliosis) sometimes experience a state of severe hypoxemia without any or only mild subjective disturbances. Organ failure is never observed in these periods.

On the other hand there are two well documented studies concerning long term oxygen therapy (LTOT) that have shown in hypoxemic COPD-patients $\left(\mathrm{pO}_{2}\right.$ lower then $55 \mathrm{~mm} \mathrm{Hg}$ ) a doubling the life expectancy under oxygen.

This contradiction can be elucidated if the influence of oxygen on the ventilation is taken into account. These study patients treated with LTOT all had more or less hypercapnia (hypoventilation) due to an overload of their respiratory pump. Oxygen reduces the ventilation (seen as hypercapnia) which leads to an unloading of the respiratory muscles. Later studies to LTOT found a positive correlation between the extent of stable hypercapnia and life expectancy.

In this article the physiopathologic background of this findings are discussed. The main parameter of the regulator for the oxygen transport is not $\mathrm{pO}_{2}$ but the oxygen content. The oxygen content multiplied by cardiac output determines the extent of oxygen delivery.

Many regulatory systems (e.g. polyglobuly or expression of oxygen resistant isoenzymes of the respiratory chain) are involved to compensate the hypoxemia associated with hypoventilation which prevents an organ threatening hypoxia. This pathophysiologic finding has important impact on intensive care medicine, which usually takes only $\mathrm{pO}_{2}$ into account for therapeutic decisions (e.g. high $\mathrm{FiO}_{2}$ and high pressure support). This sometimes leads to "overtreatment", with possible harm to the patient. 
druck als Zielgröße therapeutischer Maßnahmen (z. B. hohes $\mathrm{FiO}_{2}$, hoher Beatmungsdruck) sieht. Dieses führt nicht selten zu einer Übertherapie mit entsprechendem Schaden für die Patienten.

\section{Die überbewertete Hypoxämie}

Wenn man in Interlaken die höchste Zahnradbahn Europas zum Jungfraujoch besteigt, erreicht man in relativ kurzer Zeit etwa eine Höhe von $3500 \mathrm{~m}$. Dort herrscht ein Sauerstoffpartialdruck von $510 \mathrm{~mm} \mathrm{Hg}$, was bezogen auf Meereshöhe etwa einer Sauerstoffkonzentration von $14 \%$ entsprechen würde. Gesunde haben dort einen $\mathrm{pO}_{2}$ von etwa $55 \mathrm{~mm} \mathrm{Hg}$ entsprechend einer Sättigung von etwa $86 \%$. Obwohl sie mit diesen Messwerten die Indikation zur Sauerstofflangzeittherapie in allen Empfehlungen weltweit erfüllen würden, fühlen sich die meisten Besucher kaum beeinträchtigt. Es gibt allerdings einige, die infolge Hyperventilation mit Hypokapnie entsprechende Symptome bekommen.

Eine noch dramatischere Situation erleben wir bei vielen Patienten mit Übergewicht und oft nur leichter Obstruktion, wenn sie im Schlaf hypoventilieren. Nicht wenige dieser Patienten haben zusätzlich eine obstruktive Schlafapnoe, manche gehören aber allein nur in die Hypoventilationsgruppe. Man fasst diese Patientenentität heute als Obesitas-Hypoventilations-Syndrom zusammen. Besonders die schwereren Fälle zeigen nachts oft dramatische Entsättigungen, wie in Abb. 1 schematisch dargestellt. Die meisten Pulsoxymeter beenden ihre Messung bei einer Sättigung von $50 \%$, die neueren gehen auch darunter. In unserer Klinik haben wir einzelne Patienten, die jede Nacht eine Sauerstoffsättigung von 30\% oft über einen längeren Zeitraum aufweisen (ohne Therapie). Trotzdem sind sie am nächsten Morgen wenig kompromittiert. Würde man den Sauerstoffpartialdruck online messen, so hätten diese Patienten im ungünstigsten Falle $\mathrm{pO}_{2}$-Werte um $20 \mathrm{~mm} \mathrm{Hg}$ (Abb.2). Damit unterschreiten sie deutlich alle Empfehlungen der Notfallmedizin für eine akute Intubation mit Beatmung.

Wir haben also hier auf der einen Seite Zustände mit schwerer Hypoxämie ohne akute Beeinträchtigung der Patienten. Auf der anderen Seite wissen wir aber vor allen Dingen aus den in den 80er Jahren durchgeführten Studien zur Sauerstofflangzeittherapie (Abb. 3), dass etwa eine Verdoppelung der Lebenserwartung durch Sauerstoffgabe über $16 \mathrm{~h}$ bei diesen Patienten erreicht werden kann. Diese beiden Studien [1,2] waren bereits damals randomisiert und plazebokontrolliert, so dass sie auch nach heutigen Kriterien den höchsten Evidenzgrad haben. Es scheint also so zu sein, dass die Korrektur auch einer milden Hypoxämie einen Benefit hat. Betrachtet man jedoch die Studien zur Sauerstofflangzeittherapie genauer, so fällt auf, dass man möglicherweise bei alleiniger pathophysiologischer Betrachtungsweise der Korrektur der Hypoxämie (und deren Folgeerkrankungen wie pulmonaler Hochdruck) auf das falsche Pferd gesetzt hat.

Die erwähnten beiden Studien zur Sauerstofflangzeittherapie zeigen aber auch, dass insbesondere Patienten mit stabiler Hyperkapnie von der Sauerstofflangzeittherapie profitieren. Nachfolgestudien aus neuerer Zeit bestätigen diese Sichtweise, denn die Zunahme der Überlebenswahrscheinlichkeit ist viel strenger

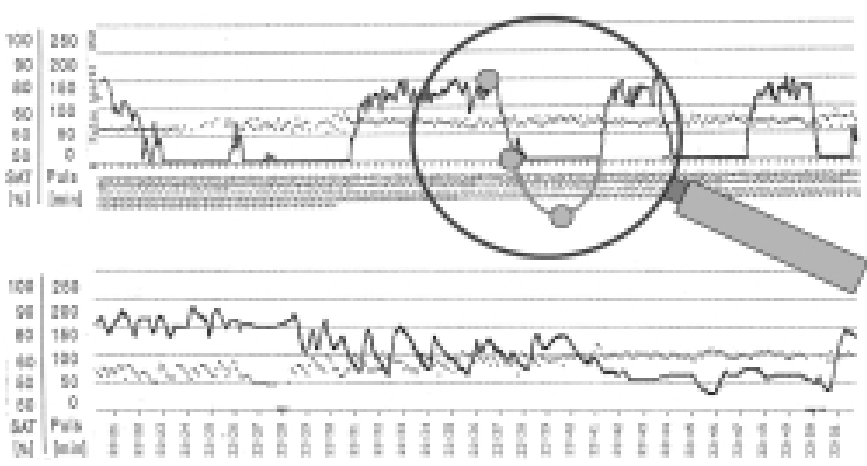

Abb. 1 Verlauf der Sauerstoffsättigung im Schlaf bei einem Patienten mit erheblichem Übergewicht und mittelgradiger chron. obstruktiver Bronchitis (Obesitas-Hypoventilations-Syndrom). Da der Sauerstoffsättigungssensor unterhalb von $50 \%$ ungenau misst, wird in diesen Bereichen die Messung nicht aufgezeichnet. Sie ist exemplarisch unter der Lupe dem wirklichen Verlauf entsprechend weitergeführt.

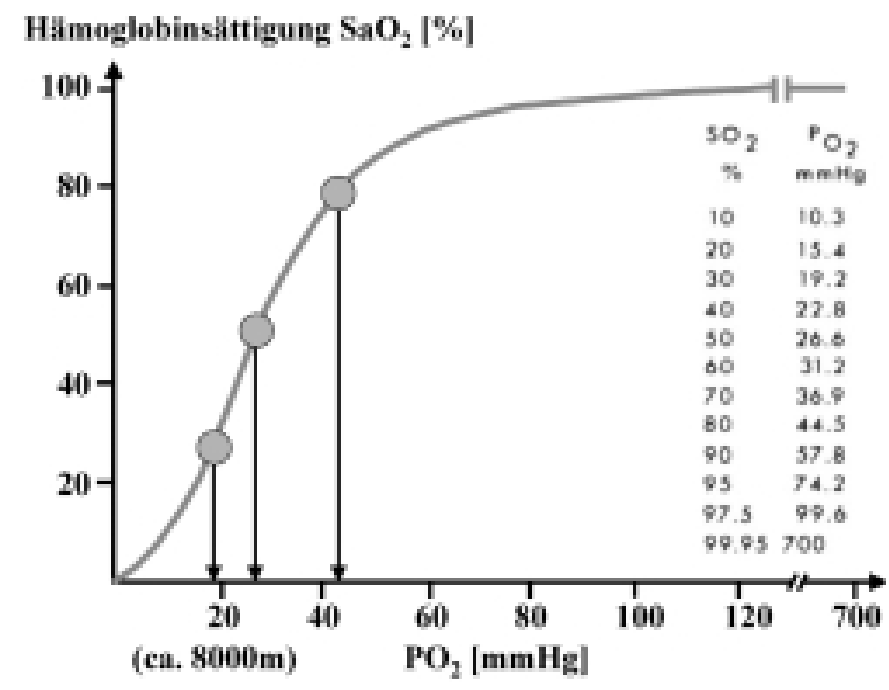

Abb. 2 Sauerstoffbindungskurve des Hämoglobins mit den drei exemplarischen Messpunkten nach Abb. 1. Angegeben ist der aus der Sättigung resultierende Sauerstoffpartialdruck.

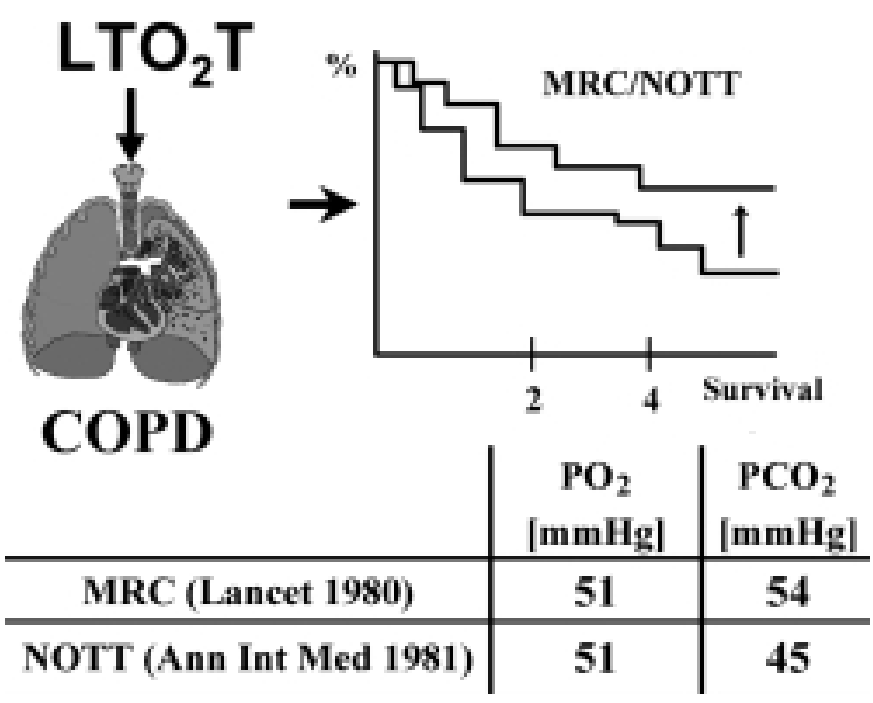

Abb. 3 Schematische Darstellung der plazebokontrollierten randomisierten Studie zur Sauerstofflangzeittherapie MRC und NOTT. Angegeben sind die Mittelwerte des Sauerstoffpartialdrucks für beide Kollektive sowie die gemessene Verdoppelung der Lebenserwartung durch die Langzeitsauerstoffgabe. Zusätzlich angegeben sind die $\mathrm{PCO}_{2}$-Werte, die in beiden Kollektiven im hyperkapnischen Bereich lagen. 
Tab. 1 Nachfolgestudien zur Sauerstofflangzeittherapie aus den letzten Jahren. Allein in der Arbeit von Gorecka et al. wurde eine Kontrollgruppe ohne Therapie verwendet. Beide Patientengruppen mit COPD und milder Hypoxämie zeigten keinen Unterschied in der Überlebensrate. Die Studien von Chailleux, Veale und Aida sind multivariate Analysen zur Sauerstofflangzeittherapie auf verschiedene Einflussvariablen. Allen Studien gemeinsam ist die Feststellung, dass eine stabile Hyperkapnie mit einer verlängerten Lebenserwartung gekoppelt ist bzw. dass die Lebenserwartung steigt, wenn die Hyperkapnie unter Sauerstofftherapie zunimmt. Sliwinski et al. hatten untersucht, ob der Einfluss der Sauerstofflangzeittherapie auf den Pulmonalisdruck prognostische Bedeutung hat. Überraschenderweise wurde das nicht beobachtet. Subjektive Einflüsse der Sauerstofftherapie wurden in der Studie von Okubadejo et al. nicht beobachtet. Näheres siehe Text

\begin{tabular}{|c|c|c|c|c|c|c|}
\hline Autor & Anzahl & $\begin{array}{l}\mathrm{pO}_{2} \\
(\mathrm{~mm} \mathrm{Hg})\end{array}$ & $\begin{array}{l}p \mathrm{CO}_{2} \\
(\mathrm{~mm} \mathrm{Hg})\end{array}$ & Parameter & Evidenzgrad & Ergebnisse \\
\hline Challeux 1996 & са. 24000 & 53 & 48 & survival & III & pCO2 $\uparrow$ unter O2 RR 0.75 \\
\hline Veale 1998 & $\begin{array}{l}1425 \\
6275\end{array}$ & $\begin{array}{l}>60 \\
<60\end{array}$ & $\begin{array}{l}44 \\
47\end{array}$ & survival & III & keine Differenz \\
\hline Sliwinski & 46 & 57 & 49 & $\begin{array}{l}\text { Pap-Abfall unter } \mathrm{O}_{2} \\
\text { auf survival }\end{array}$ & Ilb & keine Differenz \\
\hline Okubadejo 1996 & $\begin{array}{l}19 \\
17\end{array}$ & $\begin{array}{l}54 \\
63\end{array}$ & $\begin{array}{l}52 \\
46\end{array}$ & Lebensqualität & $\mathrm{lb}$ & keine Differenz \\
\hline
\end{tabular}

an das $\mathrm{pCO}_{2}$ gekoppelt als an das $\mathrm{pO}_{2}[3-8]$. Ihre wesentlichen Ergebnisse sind in Tab.1 zusammengefasst. Sauerstoffgabe führt zu einer Reduktion der Ventilation mit konsekutiver Entlastung der Atempumpe, sichtbar an einer Zunahme der Hyperkapnie. Möglicherweise führt das zu einer besseren Chance, eine Exazerbation der COPD zu überstehen, was sich deutlich in einer Zunahme der Lebenserwartung zeigen würde. Die vor einigen Jahren von Gorecka et al. [3] erschienene Studie mit einer Kontrollgruppe ohne Therapie bei geringer hypoxämischen Patienten zwischen 55 und $60 \mathrm{~mm} \mathrm{Hg}$, jedoch ohne Hyperkapnie, zeigte denn auch überhaupt keinen Überlebensvorteil für die Sauerstoffbehandlungsgruppe. Diese neuen pathophysiologischen Gesichtspunkte sind inzwischen auch in die gerade erschienenen Leitlinien zur Sauerstofflangzeittherapie in Deutschland eingeflossen [9].

Geht man nun der Frage nach, ab wann eine Hypoxie gefährlich ist, so wundert man sich, dass die aktuelle Literatur seit 1965 (Medline Recherche) praktisch nichts für die Klinik Verwertbares bietet. In den Empfehlungen zur Sauerstofflangzeittherapie werden übrigens weltweit immer nur zwei Studien dazu zitiert, und zwar die von Levine et al. [10] sowie von Krop et al. [11]. Bei der ersten handelt es sich um eine Verträglichkeitsstudie zur Sauerstofflangzeittherapie bei nur 6 hypoxämischen Patienten. Die zweite hat neuropsychologische Effekte der Sauerstofftherapie gefunden, allerdings nur bei 12 hypoxämischen Patienten, wobei 10 Parameter gemessen wurden. Beide Arbeiten hätten nach heutigen statistischen Gesichtspunkten kein positives Ergebnis gezeigt. Es ist daher erstaunlich, dass diese wichtige Frage - ab wann eine Hypoxie unter Normaldruckbedingungen relevant ist - in den letzten 35 Jahren nur sehr unzureichend untersucht worden ist. Es gibt zwar einige Studien aus der Höhenmedizin zum neuropsychologischen Einfluss von extremen Bergsteigertouren im Himalaya sowie Experimente aus Unterdruckkammern, diese sind jedoch wegen der gleichzeitigen Dekompression mit Luftembolien neben der Hypoxie nur bedingt zu verwerten $[12,13]$. Bei isobarer Hypoxämie wird dieses erst bei Sauerstoffkonzentrationen unter $5-6 \%$ beobachtet [14-17].
Eine praktische Lösung der klinisch sehr relevanten Frage, ab wann ein Sauerstoffpartialdruck kritisch wird bzw. wie die Widersprüche in den Ergebnissen zur Sauerstofflangzeittherapie zu erklären sind, ergibt sich aus einer neueren Betrachtung langjährig bekannter pathophysiologischer Zusammenhänge. Entscheidend für den Energiestoffwechsel des Organismus ist nämlich nicht der Sauerstoffpartialdruck, sondern die Zahl der Sauerstoffmoleküle. Diese Größe spiegelt sich aber nur im Sauerstoffgehalt wider. Die Beziehungen untereinander sind in der Berechnung des Sauerstoffangebotes $\left(\mathrm{DO}_{2}=\right.$ Oxygen Delivery $)$ in Abb. 4 dargestellt. Das Sauerstoffangebot errechnet sich aus der Herzleistung (z.B. cardiac index oder HZV) $\times$ Sauerstoffgehalt. Der Sauerstoffgehalt selber errechnet sich aus dem Hämoglobin $\times$ Sauerstoffsättigung $\times$ Korrekturfaktor 1,3. Erst über die Sauerstoffbindungskurve erhält man dann über die Sauerstoffsättigung den Sauerstoffpartialdruck, der üblicherweise gemessen und angegeben wird. Der Sauerstoffpartialdruck ist also nur eine tertiäre Größe (sekundär wäre die Sauerstoffsättigung). Näher an der wirklichen Sauerstoffversorgung der Mitochondrien und damit der ATP-Produktion ist die Sauerstoffsättigung; direkt proportional aber erst der Sauerstoffgehalt. Damit ist natürlich noch nichts ausgesagt über die Diffusionsstrecke in die Peripherie. Diese Größen sind jedoch schwer messbar, so dass hier kaum verwertbare Daten existieren. Allein aber die Betrachtung des Sauerstoffgehalts in der Hypoxiefrage eröffnet neue Einsichten in bekannte klinische Erfahrungen.

In Abb. 5 sind die Verhältnisse zwischen Sauerstoffpartialdruck, -sättigung und -gehalt noch einmal anschaulich dargestellt: Links ein Normalfall mit einer grenzwertig niedrigen Sauerstoffsättigung. Daneben das Beispiel einer jüngeren Frau mit Menorrhagie, die eine leichte Anämie hat. Die Blutgase sind unverändert. Der Sauerstoffgehalt ist aber durch das reduzierte $\mathrm{Hb}$ etwa $15 \%$ niedriger. Legt man nun den gleichen Sauerstoffgehalt zugrunde und nimmt beispielhaft einen Patient mit Obesitas-Hypoventilations-Syndrom (Dritter von links), so ergibt sich mit der häufig kompensatorisch vorhandenen Polyglobulie eine deutliche Erniedrigung der Sauerstoffsättigung und eine noch 
Sauerstoffangebot: $\mathrm{DO}_{2}$

\section{DO $_{2}=$ Herzminutenvolumen $\times$ Sauerstoffgehalt}

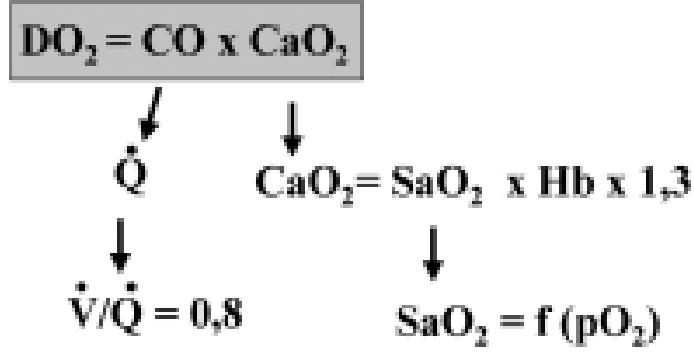

Abb. 4 Berechnung des Sauerstoffangebotes $\left(\mathrm{DO}_{2}\right)$, das der Organismus als Führungs- bzw. Stellgröße für die Regulation der Herz- und Atmungsleistung nimmt. Wesentlich dabei ist der Sauerstoffgehalt, der die Zahl der Sauerstoffmoleküle widerspiegelt. Der Sauerstoffpartialdruck ist nur eine tertiäre Größe. Die Beziehungen sind hier abgeleitet. Das Herzminutenvolumen selbst wird in Beziehung zur Ventilation als Ventilations-/Perfusionsquotient ebenfalls als zentrale Größe geregelt. Näheres siehe Text.

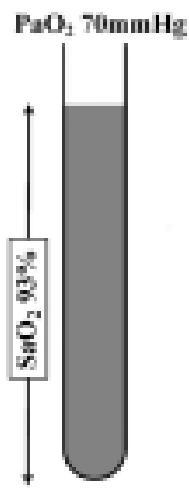

IIb $13 \mathrm{~g} \%$ $\mathrm{CaO}_{1} 16,4 \mathrm{~m} / \mathrm{s}$ Narmol

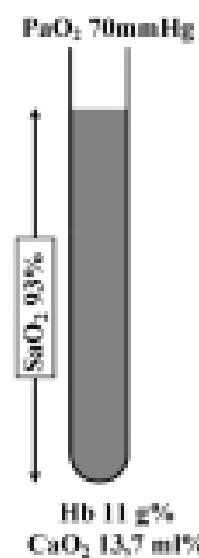

Benhorrhagive

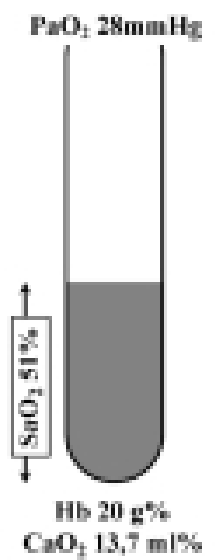

Oberins-

HypovenwilwiowsSymirow

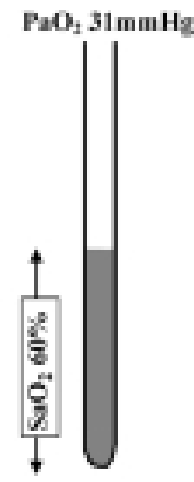

$\mathrm{Hb} 6 \mathrm{~g} \%$ $\mathrm{CaO}_{2} 4,7 \mathrm{ml}$

Skafiase + Chesshotung
Abb. 5 Anschauliche Darstellung der Beziehung zwischen Zahl der Sauerstoffmoleküle (Sauerstoffgehalt), Sauerstoffsättigung und Sauerstoffpartialdruck. Die beiden Abbildungen links zeigen den Einfluss einer leichten Anämie (Menorrhagie) auf den Sauerstoffgehalt, ohne dass sich dabei Partialdruck und Sättigung ändern. Bezieht man diese Reduktion des Sauerstoffgehaltes von 13,7\% auf ein anderes Krankheitsbild (Obesitas-Hypoventilations-Syndrom) (drittes Reagenzglas) und nimmt - wie bei diesen Patienten häufig beobachtet - eine Polyglobulie an, so zeigt sich eine dramatische Reduktion des Sauerstoffpartialdrucks. Diese Hypoxämie spiegelt sich aber nicht als Hypoxie im Gewebe wider, denn der Sauerstoffgehalt ist nur gering reduziert. Das rechte Reagenzglas zeigt einen eigenen klinischen Fall mit schwerer Anämie bei einer langsamen Ulkusblutung und zusätzlicher schwerer ventilatorischer Insuffizienz infolge einer Skoliose. Dieses Beispiel zeigt, wie weit der Sauerstoffgehalt sinken kann, ohne dass ein unmittelbar lebensbedrohlicher Zustand resultiert. Würde man diesen Sauerstoffgehalt bei einem angenommenen normalen $\mathrm{Hb}$ auf die Bindungskurve umrechnen, so käme man etwa auf Sauerstoffpartialdrücke von unter $15 \mathrm{~mm} \mathrm{Hg}$.

größere des Sauerstoffpartialdrucks. Trotzdem hat der Patient mit Obesitas-Hypoventilations-Syndrom und die junge Frau mit Menorrhagie im Prinzip die gleiche Sauerstoffversorgung in der Peripherie.
Der letzte Fall stellt eine Extremvariante aus eigener Erfahrung dar. Es handelt sich um eine Patientin mit schwerer, aber stabiler Restriktion (Skoliose), die mit nasaler Heimbeatmung eingestellt war. Sie kam in das Krankenhaus mit einer schweren Anämie infolge einer chronischen Ulkusblutung. Der Sauerstoffgehalt war dramatisch erniedrigt. Die Patientin konnte zwar nur noch wenige Schritte infolge der Luftnot gehen, hatte aber keine sonstigen Organausfälle, weder zerebral noch renal. Würde man in diesem Falle den Sauerstoffgehalt auf ein normales Hämoglobin beziehen, so wäre der Sauerstoff-Partialdruck etwa bei $10 \mathrm{~mm} \mathrm{Hg}$ gewesen.

Einige neuere Daten aus der Intensivmedizin bzw. Kardiologie unterstützen diesen Hintergrund. Wir haben zeigen können [18], dass bei Patienten mit ventilatorischer Insuffizienz und stabiler Anämie die Atemarbeit deutlich sinkt, wenn das Hb durch Blutgabe (im Mittel 2,2 Erythrozytenkonzentrate) angehoben wird. Die Sauerstoffsättigung bzw. der $\mathrm{PaO}_{2}$ ändert sich nicht. Infolge der Anhebung des Hämoglobins steigt allerdings naturgemäß der Sauerstoffgehalt. Patienten mit Anämie ohne Lungenerkrankung zeigen diese Reduktion der Atemarbeit nicht. Die Atemarbeit selbst ist ohne Ösophaguskatheter nicht genau zu messen. Sie korreliert aber im Einzelfalle ausgezeichnet mit dem Atemminutenvolumen, so dass man den Effekt auch am Rückgang des Atemminutenvolumens darstellen kann.

Eine kürzlich erschienene Arbeit von Wu et al. [19] hat 80000 ältere Patienten mit akutem Myokardinfarkt untersucht, die aus unterschiedlichen Gründen Blut bekommen haben. Dabei fand sich eine außerordentlich strenge positive Korrelation der 30-Tage-Mortalität von der Blutgabe bei entsprechender Anhebung des Hämatokrits, wenn dieser erniedrigt war. In einer anderen Studie von Silberberg et al. [20] bei Patienten mit schwerer kongestiver Herzinsuffizienz (NYHA III-IV) wurde der Effekt der Blutgabe untersucht. Dabei fand sich eine deutliche Besserung der Belastbarkeit (NYHA-Klassenrückgang von 3,8 auf 2,2), eine deutliche Reduktion der Krankenhaustage von etwa 14 auf 3 Tage sowie Rückgang des Diuretikaverbrauchs und Besserung der Nierenwerte.

Dieses alles sind indirekte Hinweise, dass dem Sauerstoffgehalt die zentrale Rolle in der Beurteilung der Energieversorgung der Organe zukommt. Was als kritische Grenze des Sauerstoffpartialdruck bei Gesunden bzw. bei Kranken mit chronischen respiratorischen Problemen anzusetzen ist, bleibt offen. Es gibt weder Sollwerte noch vernünftig verfügbare Daten aus der Literatur. Aus eigener Erfahrung kann man abschätzend sagen, dass bei vorher gesunden Patienten ein Abfall um die Hälfte kritisch werden könnte. Bei Patienten, die genug Zeit haben, Kompensationsmechanismen infolge Hypoxämie wie Polyglobulie, Veränderung der 2,3-DPG-Konzentration in den Erythrozyten, Expression von sauerstoffresistenteren Isoenzymen der Atmungskette, Aktivierung des Glukosetransportes usw. [21] zu entwickeln, ist der Wert sicher niedriger. Kritisch wird es wahrscheinlich erst, wenn ein Drittel des Normalwertes unterschritten wird.

Die vorläufigen Untersuchungen zeigen deutlich, dass der üblicherweise hergestellte Zusammenhang in Notfallsituationen zwischen einem häufig erniedrigten $\mathrm{PaO}_{2}$ und dem Allgemeinzustand des Patienten nicht mit einer Kausalität gleichzusetzen ist. Die zugrunde liegende Erkrankung führt i.d.R. zu einer Reduzie- 
rung des Allgemeinzustandes und auch häufig zu einem reduzierten $\mathrm{PaO}_{2}$. Jeder kennt aber aus der Notfallmedizin Patienten, die bei oft normalem Sauerstoffpartialdruck schwer erkrankt waren und umgekehrt. Daher sollte auch in der akuten Notfallsituation angestrebt werden, die Pathophysiologie der aktuellen Situation zu ergründen und die daraus relevanten therapeutischen Schlüsse zu ziehen. Wird das konsequent durchgesetzt, kann man oft feststellen, dass üblicherweise zu viel Aufwand getrieben wird, um den Sauerstoffpartialdruck anzuheben. Da Sauerstoff ab $40-45 \%$ im Inspirationsfluss zu einem Sistieren der mukoziliären Clearance und bei höheren Konzentrationen zu einer erhöhten Leckage des respiratorischen Epithels führt, werden dadurch Folgeerkrankungen wie Atelektase, Pneumonie und insbesondere Schocklunge begünstigt. Unter Berücksichtigung eines ausreichenden Sauerstoffgehaltes ist es daher oft gar nicht erforderlich, entsprechend viel Sauerstoff zu applizieren bzw. bei beatmeten Patienten die Beatmungsdrucke unnötig zu erhöhen, um z.B. eine $\mathrm{PaO}_{2}$ über $80 \mathrm{~mm} \mathrm{Hg}$ oder gar eine Sättigung von $95 \%$ zu erreichen - wie es in vielen Lehrbüchern der Anästhesiologie noch vermerkt ist. Dieses erzeugt bei nicht wenigen Patienten mehr Schaden als die zugrundeliegende Erkrankung, die zur Krankenhausaufnahme geführt hat.

\section{Literatur}

${ }^{1}$ Nocturnal Oxygen Therapy Trial Group. Continuous or nocturnal oxygen therapy in hypoxemic chronic obstructive lung disease: a clinical trial. Ann Intern Med 1980; 93: 391 - 398

2 Report of the Medical Research Council Working Party. Long term domiciliary oxygen therapy in chronic hypoxic cor pulmonale complicating chronic bronchitis and emphysema. Lancet 1981; 28;1: 681-686

${ }^{3}$ Gorecka D, Gorzelak K, Sliwinski P et al. Effect of long-term oxygen therapy on survival in patients with chronic obstructive pulmonary disease with moderate hypoxaemia. Thorax 1997; 52: 674-679

${ }^{4}$ Aida A, Miyamoto K, Nishimura M et al. Prognostic value of hypercapnia in patients with chronic respiratory failure during long-term oxygen therapy. Am J Respir Crit Care Med 1998; 158: 188-193

${ }^{5}$ Chailleux E, Fauroux B, Binet $\mathrm{F}$ et al. Predictors of survival in patients receiving domiciliary oxygen therapy or mechanical ventilation. A 10-year analysis of ANTADIR Observatory. Chest 1996; 109: 741 - 749

${ }^{6}$ Okubadejo AA, Paul EA, Jones PW et al. Does long-term oxygen therapy affect quality of life in patients with chronic obstructive pulmonary disease and severe hypoxaemia? Eur Respir J 1996; 9: 2335-2339
${ }^{7}$ Sliwinski P, Hawrylkiewicz I, Gorecka D et al. Acute effect of oxygen on pulmonary arterial pressure does not predict survival on long-term oxygen therapy in patients with chronic obstructive pulmonary disease. Am Rev Respir Dis 1992; 146: 665-669

${ }^{8}$ Veale D, Chailleux E, Taytard A et al. Characteristics and survival of patients prescribed long-term oxygen therapy outside prescription guidelines. Eur Respir J 1998; 12: 780-784

${ }^{9}$ Magnussen H, Goeckenjan G, Kohler D et al. Leitlinien zur Sauerstofflangzeittherapie. Pneumologie 2001; 55: 454-464

${ }^{10}$ Levine BE, Bigelow DB, Hamstra RD et al. The role of long-term continuous oxygen administration in patients with chronic airway obstruction with hypoxemia. Ann Intern Med 1967; 66: 639-650

11 Krop HD, Block AJ, Cohen E. Neuropsychologic effects of continuous oxygen therapy in chronic obstructive pulmonary disease. Chest 1973; 64: $317-322$

${ }^{12}$ Abraini JH, Bouquet C, Joulia $\mathrm{F}$ et al. Cognitive performance during a simulated climb of mount everest: implications for brain function and central adaptive processes under chronic hypoxic stress. Pflugers Arch 1998; 436: 553-559

${ }^{13}$ Nicolas M, Thullier-Lestienne F, Bouquet $C$ et al. A study of mood changes and personality during a 31-day period of chronic hypoxia in a hypobaric chamber (Everest-Comex 97). Psychol Rep 2000; 86: $119-126$

${ }^{14}$ Cervos Navarro J, Kunas RC, Sampaolo S et al. Heart mitochondria in rats submitted to chronic hypoxia. Histol Histopathol 1999; 14: $1045-1052$

${ }^{15}$ Halperin FA, Cheema-Dhadli S, Chen CB et al. Alkali therapy extends the period of survival during hypoxia: studies in rats. Am J Physiol 1996; 271: R381-R387

${ }^{16}$ Kaijser L, Grubbstrom J, Berglund B. Myocardial lactate release during prolonged exercise under hypoxaemia. Acta Physiol Scand 1993; 149: 427-433

${ }^{17} \mathrm{Kamel} \mathrm{KS}$, Mazer CD. Effect of $\mathrm{NaHCO}_{3}$ on cardiac energy metabolism and contractile function during hypoxemia. Crit Care Med 2001; 29: $344-350$

${ }^{18}$ Schönhofer B, Wenzel M, Geibel M et al. Blood transfusion and lung function in chronically anemic patients with severe chronic obstructive pulmonary disease. Crit Care Med 1998; 26: 1824-1828

${ }^{19} \mathrm{Wu}$ WC, Rathore SS, Wang Yet al. Blood transfusion in elderly patients with acute myocardial infarction. N Engl J Med 2001; 25; 345: $1230-1236$

20 Silverberg DS, Wexler D, Sheps D et al. The effect of correction of mild anemia in severe, resistant congestive heart failure using subcutaneous erythropoietin and intravenous iron: a randomized controlled study. J Am Coll Cardiol 2001; 37: 1775-1780

${ }^{21}$ Köhler D, Schönhofer B. Apnoe-Hypopnoe: Eine oder zwei Entitäten? Pneumologie 1998; 52: $311-318$ 